\title{
Piezoceramic Ultrasound Transducer Enabling Broadband Transmission for 3D Scene Analysis in Air
}

\author{
Kellner Johannes, Schweinzer Herbert \\ Institute of Electrical Measurements and Circuit Design, Vienna University of Technology \\ Gusshausstrasse 25/354, 1040 Vienna, Austria
}

\begin{abstract}
Frequency-modulated ultrasound signals like chirp-signals show variations in phase at the different transmission angles. Analyzing the phase of ultrasound chirp-echoes enables to accurately determine distance and polar angle to the point of reflection. Though single piezoceramic ultrasound transducers have a small bandwidth, an ultrasound transducer made of a combination of piezoceramic elements with different resonance frequencies as presented in this work enables transmission of broadband chirpsignals and shows the required rotational symmetry.
\end{abstract}

\section{Introduction}

Ultrasound transducers play an important role in imaging under water, medical imaging and nondestructive material testing (NDT). Though air ultrasound systems are found in several fields, e. g. for gas flow measurements, robot navigation and fill level measurements, they won only in car industry importance applied as parking help. Air ultrasound systems have the advantage that distances to reflecting objects can be measured in dusty, dirty or smoky environments [8], where optical sensors would fail. In literature different kinds of ultrasound 3D positioning and scene analysis systems can be found using A-B measurements (receiver placed on the object whose position is of interest) [1, 2], others use reflective measurements [3], but all use only the time of flight (TOF) information of the ultrasound signals. Our group is researching the phase information of echo signals coming from reflecting objects.

Frequency-modulated chirp-signals transmitted by ultrasound sensors with broad bandwidth show different variations in phase at different directions of the radiated sound pattern. With the phase information of the echo signal it is possible to determine the angle of the point of reflection with respect to the main transmission direction of the ultrasound transducer, representing the polar angle in a spherical coordinate system with the main transmission direction being the $z$ axis. Processing the frequency-modulated chirp-signal by use of a correlation algorithm delivers high accuracy of the distance measurement. Using only one ultrasound transducer transmitting a broadband signal and assuming rotational symmetry, we get the distance and one of the two angles needed to exactly determine the 3D coordinates of the reflection point, instead of getting only the distance like in other known systems.

Off the shelf ultrasound transducers for air applications are based on different technologies, like electrostatic transducers, PVDF (Polyvinylidene fluoride) transducers, piezoceramic and piezocomposite transducers. Although typical constructions of electrostatic transducers are not well suited for industrial environment, many air ultrasound applications are based on this transducer type producing high sound pressure amplitudes by simultaneous small power consumption. Though electrostatic transducers have also an advantageous broad bandwidth, they are not appropriate to be used for polar angle measurements, because they still cannot be produced with a good rotational symmetry. The only air ultrasound transducers that show appropriate rotational symmetry are piezoceramic transducers. Piezoceramic transducers have a narrow bandwidth and are driven most effectively at their geometry-dependent resonance frequencies.

Our idea to arrange piezoceramic components with different resonance frequencies in a transducer $[4,5,6]$ leads to two innovative advantages. On the one hand, a transmission of broadband signals having reproducible rotational transmission symmetry is enabled, on the other hand an activation of every single element of the ultrasound transducers transmitting surface can be controlled by simply changing the driving frequency, which leads to frequency controlled radiation patterns and increased changes of the transmission angle dependent signal phases and therefore to a better angle detectability by echo signal processing. 


\section{Three Ring Annular Design}

The piezoceramic components are bonded to each other using soft silicone rubber (GE silicones RTV12), which on the one hand adheres well on the components and on the other hand mechanically decouples the single piezoceramic components. Various designs of combined piezoceramic components are imaginable. Some were already analyzed using simulation models. A few prototypes were built and their vibration and transmission behaviour investigated.

The transducer studied in this paper is shown in Fig. 1 and consists of three interleaved piezoceramic annular rings made of soft-PZT PIC151 from PI Ceramic GmbH, Germany. The rings were manufactured at our institute by grinding piezoceramic bulk material. The radial geometric dimensions (outer diameter, inner diameter) of the piezoceramic rings were a compromise between necessary transmitting surface and a maximum of wanted overall dimensions of the transducer configuration. The length dimensions of the rings were fitted to get uniformly distributed resonance frequencies in a range from $74 \mathrm{kHz}$ to $92 \mathrm{kHz}$ (see Table 1). The upper frequency bound is given by the maximum frequency of the separated receiver microphone (see later in this work) and the lower frequency bound results of not wanting to much distance between the single resonance peaks (see Fig. 2).

Table 1. Geometrical dimensions and resonance frequencies of the piezoceramic rings

\begin{tabular}{|c|c|c|c|c|}
\cline { 2 - 5 } \multicolumn{1}{c|}{} & Outer diameter & Inner diameter & Length & Resonance frequency \\
\hline Ring 1 & $16.1 \mathrm{~mm}$ & $5.3 \mathrm{~mm}$ & $16.8 \mathrm{~mm}$ & $74 \mathrm{kHz}$ \\
\hline Ring 2 & $25.8 \mathrm{~mm}$ & $18.1 \mathrm{~mm}$ & $19.3 \mathrm{~mm}$ & $83 \mathrm{kHz}$ \\
\hline Ring 3 & $35 \mathrm{~mm}$ & $23 \mathrm{~mm}$ & $16.5 \mathrm{~mm}$ & $92 \mathrm{kHz}$ \\
\hline
\end{tabular}

The transmitting areas of all three piezoceramic rings build a plane and are contacted with silver ink electrodes, as well as their opposite sides. In the centered hole of Ring 1, the separated receiver microphone is installed (see Fig. 5.b) again using silicone rubber as filling material to mechanically decouple the microphone from Ring 1 . Figure 2 shows the sound pressure spectrums of the three rings measured at a driving voltage of $300 \mathrm{Vpp}$. in a distance of $0.5 \mathrm{~m}$ in main transmission direction.

\section{Measuring Signal}

For both, measuring accurate distances as well as measuring the polar angle of reflecting points, chirp-signals with linearly increasing frequency are used. The bandwidth of the transducer configuration enables an effectively transmission of chirp-signals with frequencies from $68 \mathrm{kHz}$ to $108 \mathrm{kHz}$. The transmitted ultrasound signal results of the superposition of portions of the chirp-signal radiated from each of the three piezoceramic rings. A transmitted chirp-signal with duration of $0.5 \mathrm{~ms}$ is shown in Fig.3.

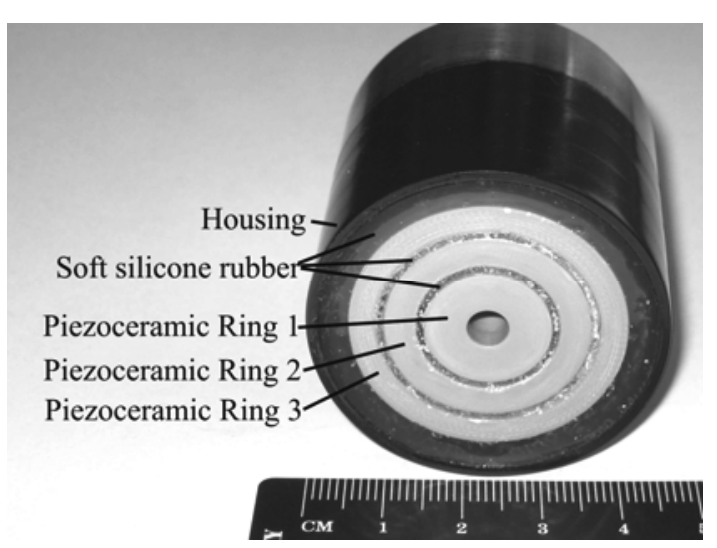

Fig. 1. Air ultrasound transducer consisting of three interleaved piezoceramic rings, each having another resonance frequency, bonded together with soft silicone rubber.

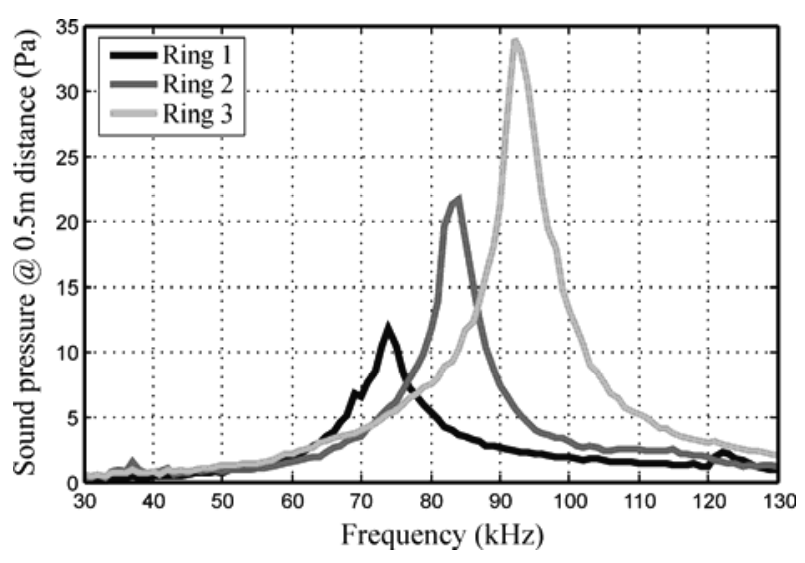

Fig. 2. Sound pressure spectrums of the 3 piezoceramic rings at $300 \mathrm{Vpp}$ driving voltage, measured in a distance of $0.5 \mathrm{~m}$. 


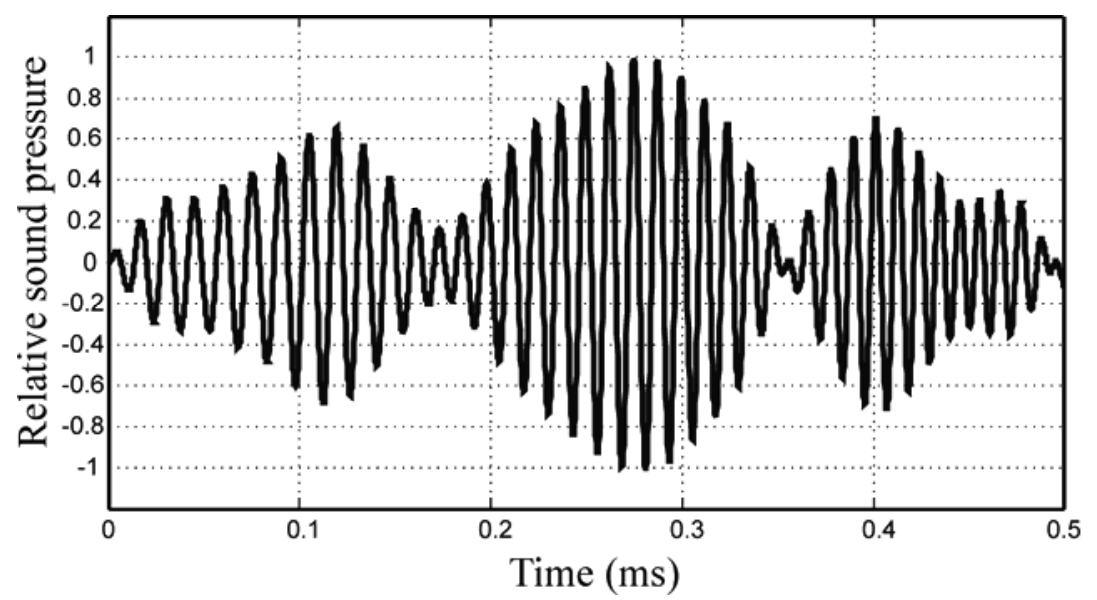

Fig. 3. Transmitted chirp-signal. Linearly increasing frequency from $68 \mathrm{kHz}$ to $108 \mathrm{kHz}$. Duration $0.5 \mathrm{~ms}$.

It can be seen, that the envelope of the transmitted signal proceeds according to the resonance peaks of the three piezoceramic rings.

\section{Measuring Method and Rotational Symmetry}

Immediately after transmitting a chirp-signal, the separated receiver microphone is enabled scanning for echoes. Once an echo is detected, the echo signal is correlated with reference signals, each reference signal according to another polar angle. The highest correlation result is scored when the echo signal is correlated with a reference signal of that polar angle that fits the angle, the echo is coming from. The accurate distance to the point of reflection is determined in parallel with the results of the polar angle measurements.

Applying this method, the polar angle detectability was analyzed at azimuth angles stepwise from $0^{\circ}$ to $330^{\circ}$ (angle steps $30^{\circ}$ ). Fig. 4 shows the result of the rotational symmetry analysis.

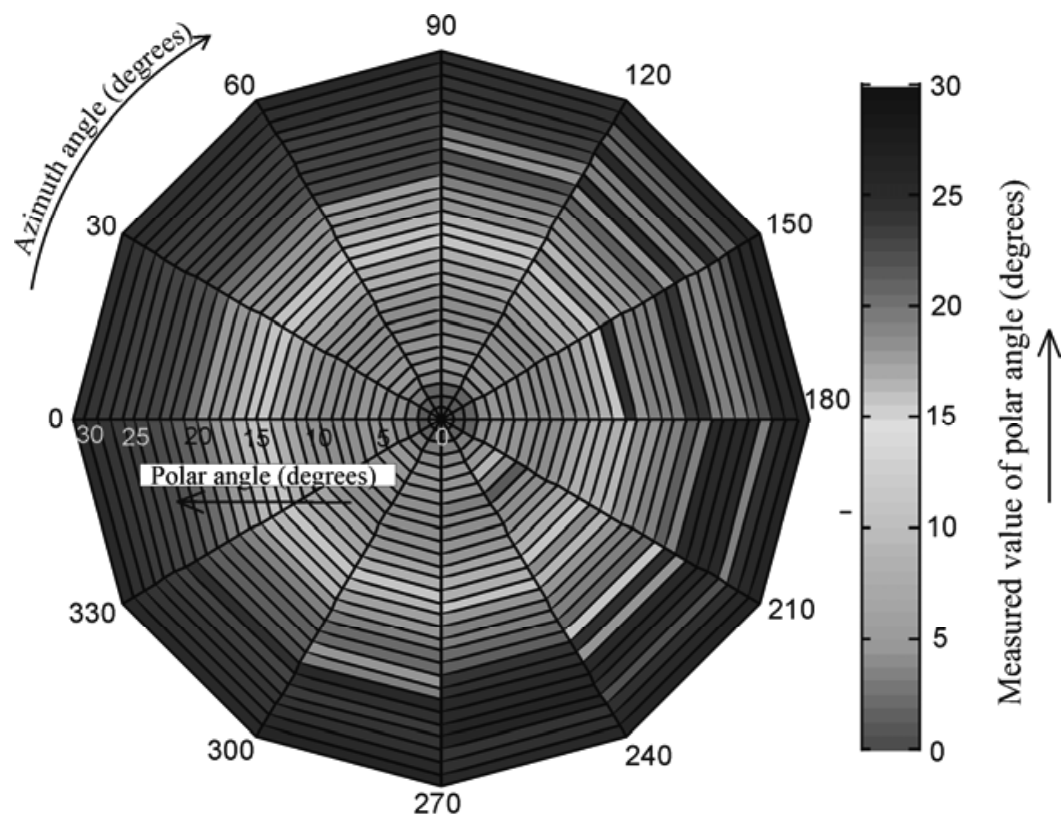

Fig. 4. Analyzing rotational symmetry of the polar angle dependent phase information stepwise from $0^{\circ}$ to $330^{\circ}$. Errors in the larger polar angles are caused by low SNR.

When measuring the polar angle, errors occur mainly at angles larger than $20^{\circ}$ where signal-tonoise-ratio (SNR) is significantly lower than in main direction. 


\section{Measuring position and orientation of a $30 \mathrm{~mm}$ diameter rod}

The annular transducer with the separated centered receiver microphone (= annular sensor) was mounted on a 2-axes-wrist / linear drive combination (PowerCube robot device) as can be seen in Fig.5.

a)

b)

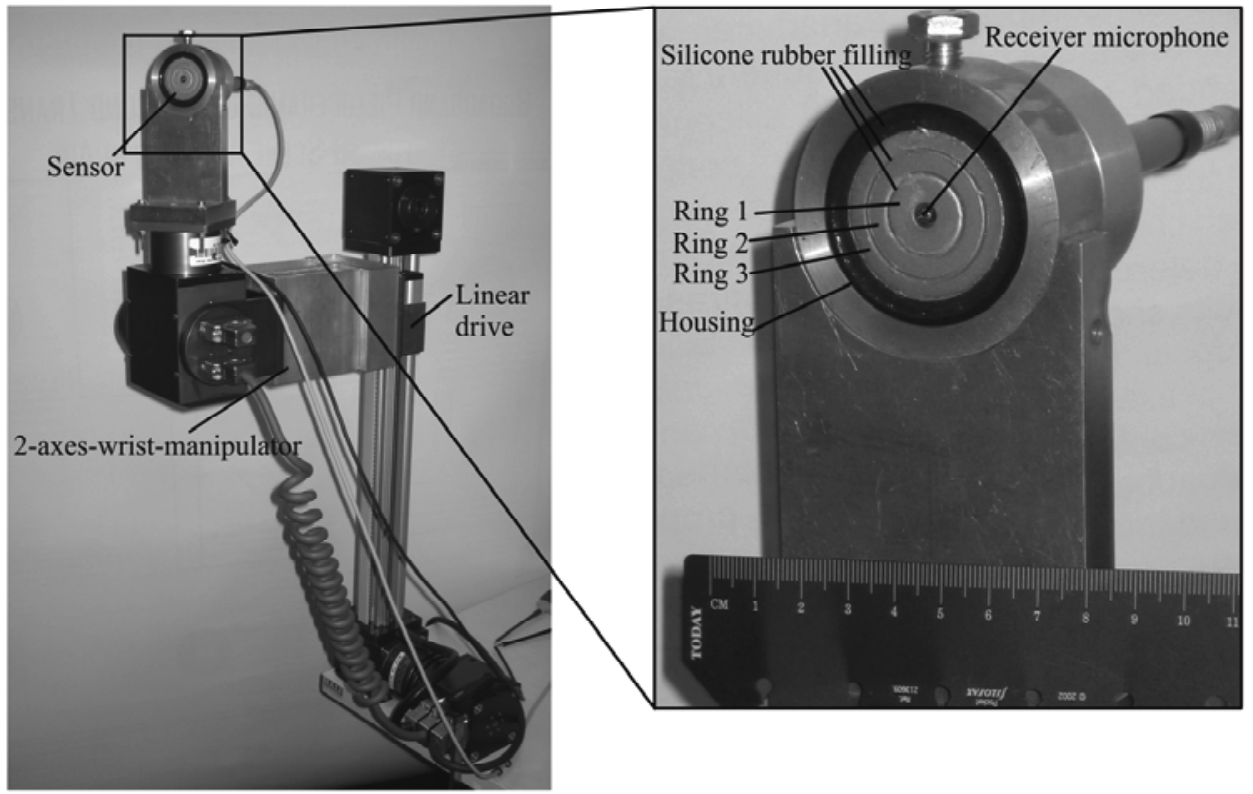

Fig. 5. a,b) Annular transducer with separated receiver microphone mounted on a PowerCube robot device (2axes-wrist-manipulator combined with a linear drive). First practical application of the annular transducer was measuring distance and polar angle to reflecting objects.

This robot device was used to move the annular sensor in the room, measuring distances and polar angles to reflecting points, all lying on a round rod with a diameter of $30 \mathrm{~mm}$ (see Fig. 6).

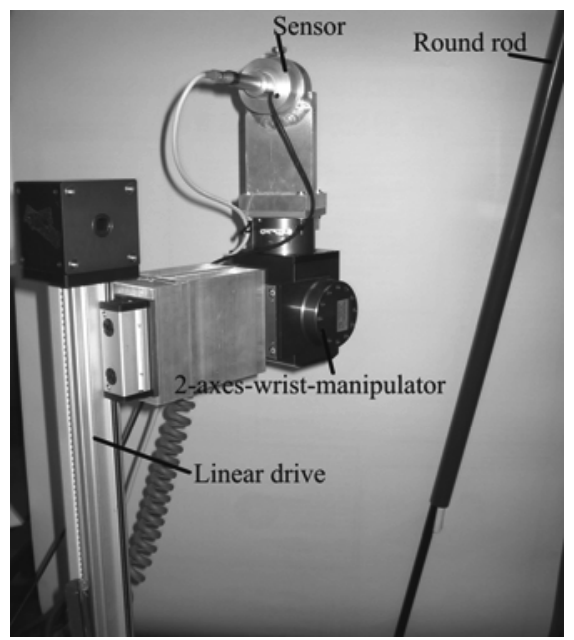

Fig. 6. Annular sensor while moving through the room and measuring reflections of a round rod.

Knowing the polar angle of the reflection point means in other words, that all possible echo rays form a cone symmetrically positioned in the direction of the sensor axis. Thus, together with the measured distance, all possible reflection points form a circle symmetrically positioned around the sensor axis (see Fig. 7). The orientation of this circle is determined by the sensor axis. Its position is determined by the measured distance between reflection point and transducers transmitting surface. The radius is given from the opening angle of the cone which is the measured polar angle. The measuring results are fully spatially determined "contact circles". 


\section{a) Rod nearly in parallel to the axis of the linear drive.}

In the first measuring scene, the sensor was moved along the linear drive nearly in parallel to the axis of the round rod. Fig. 7 shows the measured contact circles of reflecting points of the rod at 11 measuring positions with distances of $30 \mathrm{~mm}$ between each position.

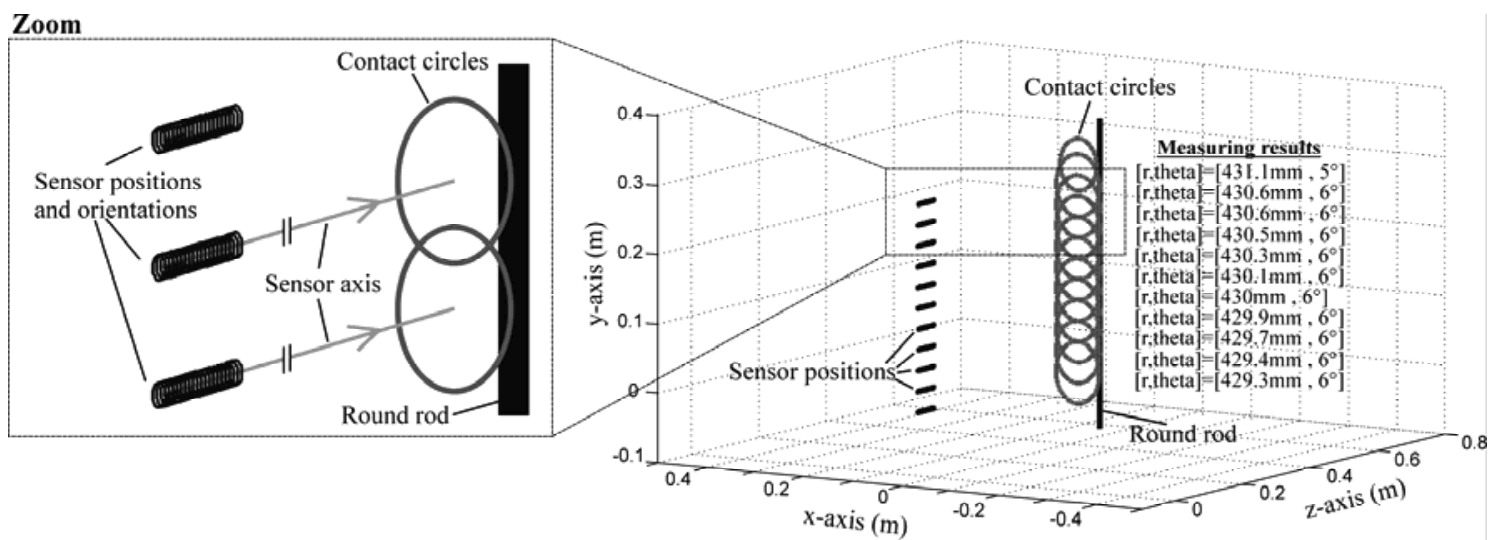

Fig. 7. Measuring scene 1. Sensor moved along linear drive axis measuring distances and polar angles to a round rod positioned nearly in parallel to the linear drive axis.

In the list of measuring results where $\mathbf{r}$ is the measured distance between sensor and reflection point on the rod, and theta is the measured polar angle to the reflection point, it can be seen that the rod axis and the moving axis of the sensor are not perfectly in parallel, because of the descending distance values in the measuring results.

\section{b) Rod not in parallel to the axis of the linear drive}

In the second measuring scene, the rod was lying diagonally in the room with respect to the linear drive axis. The sensor was again moved along the linear drive. At 31 positions with $10 \mathrm{~mm}$ space between each position the distances $\mathbf{r}$ and polar angles theta to the round rod were measured delivering the results for the contact circles shown in Fig. 8.

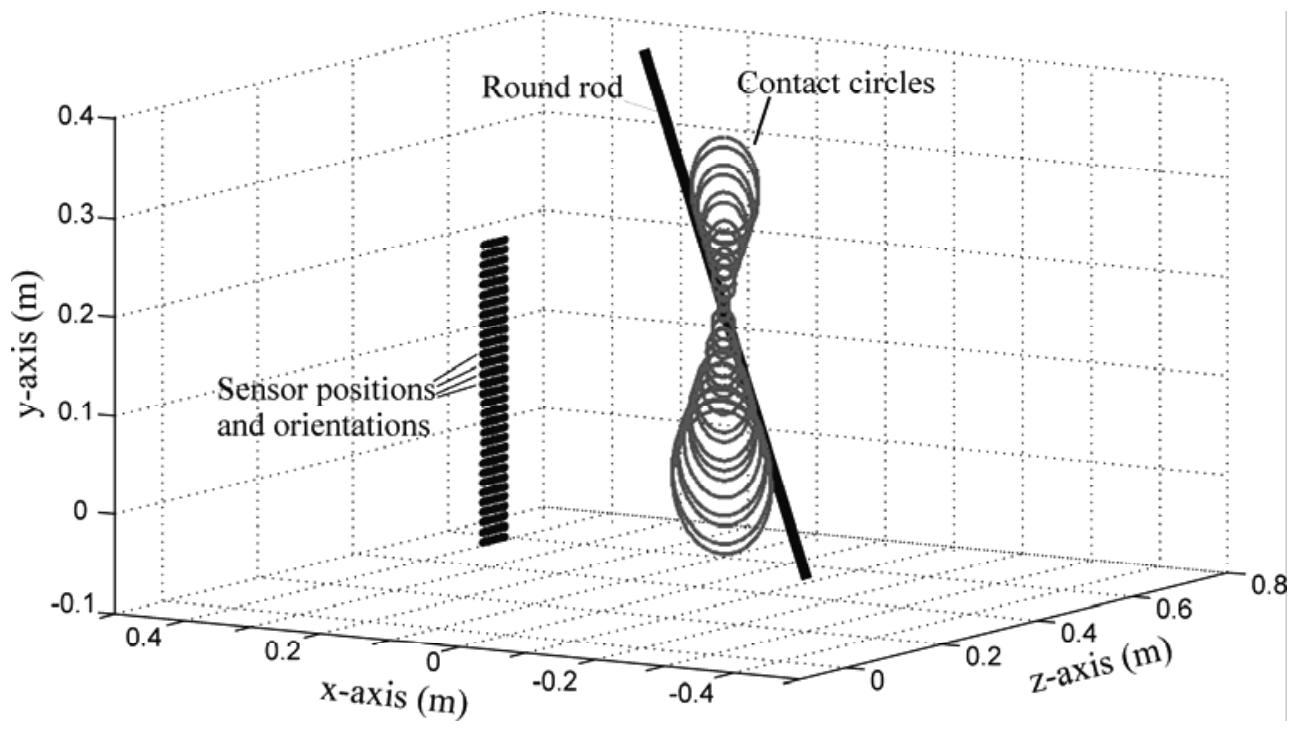

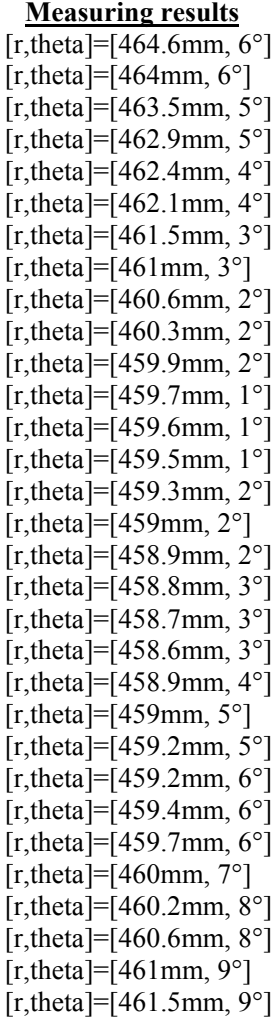

Fig. 8. Measuring scene 2. Sensor moved along linear drive axis measuring distances and polar angles to a round rod positioned diagonally in the room with respect to the linear drive axis. 
In [7] it was shown that orientation and position of an edge can be fully spatially determined measuring polar angle and distance of two reflection points lying on the edge. In principle, measuring may be performed at two arbitrary sensor positions. The algorithm in [7] is based on the knowledge that the reflection points lie on one edge.

A round rod in the room being the only reflective object, acts like an edge where again position and orientation can be calculated using the algorithms of [7] after measuring distance and polar angle of at least two reflection points lying on the rod. Ambiguous solutions of determined position and orientation of the rod can be avoided, when the sensor is moved in paths that deviate from straight lines. Measuring distance and angle of more than two reflection points leads to an overestimated system of equations helping to lower the failure possibility and to classify the measured object with respect to its type.

\section{Conclusion}

Though piezoceramic transducers have a narrow bandwidth, a combination of piezoceramic components with different resonance frequencies building one ultrasound sensor as presented in this work, allow the transmission of broadband signals like chirp-signals that can be used to accurately measure distances and polar angles to reflecting objects. Former problems of measuring polar angles in air like low accuracy when using amplitude information [7] or small detectable angle areas are not given using a transducer configuration like presented in this work.

\section{References}

[1] Mahajan, A., Walworth, M., 3D Position Sensing Using the Differences in the Time-of-Flights from a Wave Source to Various Receivers. IEEE Trans. Robot. Autom., Vol. 17, No. 1, Feb. 2001, pp. 91-94.

[2] Dijk, E. O., (Kees) van Berkel, C. H., Aarts, R. M., van Loenen, E. J., 3-D Indoor Positioning Method using a Single Compact Base Station. Proc. IEEE PERCOM'04, 2004, pp. 101-110.

[3] Capineri, L., Fiorillo, A. S., Rocchi, S., Low Frequency PVDF Transducers for 3D Object Profiling in Air. Proc. IEEE Ultrason. Symp. 1995, pp. 901-904.

[4] Kellner, J., Schweinzer, H., Piezoceramic Ultrasonic Transducer with Frequency Controlled Radiation Pattern. Proc. IEEE Instr. \& Meas. Techn. Conf., 2007.

[5] Kellner, J., Schweinzer, H., 1-3 Piezofiber - Silicone Rubber - Composite with Different Resonance Frequencies Enabling Frequency Controlled Shapes of the Ultrasound Radiation Pattern. Proc. IEEE Ultrason. Symp. 2007, pp. 1701-1704.

[6] Kellner, J., Schweinzer, H., Broadband Piezoceramic Ultrasound Transducers for 3D Scene Analysis in Air. Tagungsband zur Informationstagung Mikroelektronik 08 (ME2008), Wien, 2008, pp. 73-77.

[7] Krammer, P., Spatial Localization of Elementary Geometric Shapes with Industrial Ultrasonic Sensors. Dissertation, Institute of Electrical Measurements and Circuit Design, Vienna University of Technology, 2005.

[8] Mágori, V., Ultrasonic Sensors in Air. Proc. IEEE Ultrason. Symp. 1994, pp. 471-481. 